Monatsschrift f. Geburtshülfe u. Gynäkologie 1922;59:238-244

\title{
V. Literatur-Verzeichnis
}

\section{Geburtshilfe.}

Abels, Über die Wichtigkeit der Vitamine für die Entwicklung des menschlichen fötalen und mütterlichen Organismus. Klin. Woch. 8. IX.

S. 1785. Bauer, W., Über artefizielle Glykosurie Saccharo in der Schwangerschaft.

Z. f. G. 2. IX. S. 1413. Benthin, Die Grundlagen der künstlichen

Schwangerschaftsunterbrechung.

Th. d. Geg. 1921. H. 9. Björkenheim, Mund- und Zahnkrankheiten während der

Schwangerschaft.

Munch, med. Woch. 21. IV. S. 597. Blair Bell, Einige alltägliche, doch oft unerkannte

geburtshilfliche

Schwierigkeiten. Brit. m. J. Nr. 3171. Brodhead uncl Kassebohm, Mola hyclatitosa: 10 Fälle.

Am. J. obst. gyn.

IV. 1. S. 45. Bumm, Behandlung der Eklampsie. Munch, med. Woch. 15. IX. S. 1351. Calkins, Morphologie des menschlichen Fötus mit Beziehung des geburts-

hilflichen Messen des Ivopfes. Am. J. obst. gyn. IV. 2. S. 109. Campbell, Die Lage des Arztes einem kriminellen Abort gegenüber. Br. $\mathrm{m}$.

J. Nr. 3180. Cornell, Verfahren, um bei der Sectio caesarea alle Flüssigkeit aufzusaugen.

Am. J. obst, gyn. IV. 2. S. 182. Cosbie, Syphilis und Abort. Totgeburt und Kindersterblichkeit. Am. J.

obstet. gyn. IV. 1. S. 40.

Literatur-Verzeichnis.

239

Crawford, Spasmodische Uterusstriktur. Brit. in. J. Nr. 3187.

Dantín, Fall von Pyaemia puerperalis chr. - schließlich durch Vakzine-

therapie geheilt. Gyn. obst. VI. 1. S. 37. Deluca, Über den symptomatischen Icterus neonatorum. Munch, med.

Woch. 25. VIII. S. 1254. Dewes, Über den Schulterschmerz als Symptom des äußeren

Fruchtkapsel-

aufbmches. Z. f. Gyn. 6. IX. S. 1482. Dietrich, Behandlung fieberhafter Aborte. Klin. Woch. 2.

IX. S. 1798. Döderlein, II. Anklage gegen eine Hebamme wegen fahrlässiger Tötung.

Munch, med. Woch. 11. VIII. S. 1191. Döderlein, III. Anzeige gegen einen praktischen Arzt wegen fahrlässiger

Körperverletzung. Munch, med. Woch. 8. IX. S. 1308. Eberle, Spontaner Scheidenabriß während der Gebtirt, nebst Berrierkungen

zur Uterusruptur. Schweiz. med. Woch. 1921. Xr. 37. Ehrenfest, Bessere Geburtshilfe und das

Problem der Geburtsschädigung

der Neugeborenen. Am. J. obst. gyn. IV. 1. S. 61. Eisenreich, Allerlei aus der operativen

Geburtshilfe. Munch, med. Woch.

8. IX. S. 1306. Ellerbroek, Zur operativen. Behandlung des Xabelschmirbruches. Dtsch. 
med. Woch. 8. IX. S. 1213. Fríedländer, Die Behandlung der Fluor mit Thyoparametron. Dtsch. med.

Woch. 25. VIII. S. 1139. Gänßle, Klinisches über die Fahräussche Schwangerschaftsreaktion. Munch, med. Woch. 21. IV. S. 578. Gold, Fin neuer Aspirator. Z. f. Gxn. 26. VIII. S. 1380. Goldberger, Kurzer Überblick über die physiologische und embryologische Genesis des Pseudohermaphroditismus. Am. J. obst. gyn. IV. 2. S. 130. Gra†fagnino, Die in dem Chariteekrankenhaus von New-Orleans becbachteteu

Extrauterinschwangerschaften. Am. J. obst. gyn. IV. 1. S. 71. (iraffagnino, Graviditas extrauterina. Am. J. obst. gyn. Xr. 2. S. 148. Grätzer, Ein Beitrag zur Abortbehandlung. Med. Klin. 27. VIII. S. 1118. Halm und Friedmann, Über schonende Alkoholvakuumsterilitation fur Instrumente. Katgutt usw. Dtsch. med. Woch. 25. VIII. S. 1125. Iíaipèr, Bandlscher Ring und Geburtserschwerung. Surg. gyn. obst.

Aug. 22. S. 148. Harris, Schwangerschaft und Geburt bei jüngeren Primiparen. Johns

Hopk. Hosp. bull. Xr. 371. Herrick, Schwangerschaft und Herzkrankheit vom InternistenStandpunkt

aus. Am. J. obst. gyn. IV. 1. S. 1. Hellmuth, Statistische Beiträge zur Frage der

Geschlechtsbildung des

Kincles. Z. f. Geb. u. Gyn. LXXXIV. S. 74. Hinselmann, Einiges über die Unterbrechungen der Kapillarströmung bei

Schwangeren. Z. f. G. 9. IX. S. 1426. Hoeck, Ein Beitrag zur Frage der Gebärmutterzerreißung unter der Geburt

ohne ersichtliche Ursache. Z. f. G. 20. VIII. S. 1378. Klein, P., Eine neue Methode zur Anregung der Wehentätigkeit. Z. f. G.

12. VIII. S. 1308. Kleinknecht, Ein kleiner Hilfsapparat bei der Blutdr«ckmessung am

Menschen. Munch, med. Woch. 21. IV. S. 592.

240

Literatur-Verzeichnis.

Knopf, Über einen Fall von Verwachsung der kleinen Labium post partum.

Wien. klin· Woch. 24. VIII. S. 715. Kratochril, Pathologisch $\beta$ Anwachsung der Placenta mit SpontanruptHides graviden Uterus. Cas. lék. cesk. 1921. Nr. 47. Kritzler, Zur Frage der ,, Reform" des geburtshilflichen Studiums. Z. f. G.

16. IX. S. 1487. Küstner, H., Künstlich erzeugte Glykosurie und ihre Bewertung für die

Frühdiagnose der Gravidität in der Praxis. - Hellmuth, Erwiderung.

Med. Klin. 26. VIII. S. 1747. I^epmann, W., Violente Verletzungen bei der Behandlung des

Abortes

in bildlicher Darstellung. Med. Klin. 27. VIII. S. 1110. Liepmann, W., Der geburtshilfliche

Phantomkurs. Urban und Schwarzen-

berg. Berlin 1922. 2. Aufl. Linzenmeyer, Zur Deutung der Kapillarstasen. Z. f. G. 9. IX. S. 1428.

Mathes, Das Ödem der Schwangeren. Z. f. Geb. u. Gyn. LXXXIV. 1.

S. 26. Müller, Arthur, Die Mechanik der Geburt, Z. f. G, 26. VIII. S. 1361. Neis, Inversio uteri

puerperalis totalis. Z. f. G. 2. IX. S. 1421. Nürnberger, Zur Biologie des Isthmus uteri. Z. f. Geb. u. Gyn. LXXXV.

H. 1. S. 1. Ottow, Schwangerschaft nach doppelseitiger Sactosalpinx mit Douglas-

abszeß. Z. f. G. 2. IX. S. 1406. J'íc Pheeters, Verhütung von Striae gravid. Rektum-Diastase, Viszeroptosis

und Ptosis der Brüste während der Schwangerschaft. Am. J. obst. 
gyn. IV. 2. S. 178. Mc Pherson, Über Schwangerschaftstoxämie als Eklampsie. Am. J. obst. gyn. IV. 1. S. 50. Portner, Erkrankungen der Harm•òhre. Med. Klin. 27. VIII. S. 1122. Potocki und Lelièvre, Chorionepithelioma malignum nach Mola hydatiformis mit Scheiden- und Lungenmetastasen. Gynec. obst. VI. 2. S. 112. Reichert, Zur Behandlung des Puerperalfiebers. Dtsch. med. Woch. 18. VIII. S. 1102. Reichert, Geburtenzahl im Regierungsbezirk Düsseldorf. Dtsch. med. Woch. 8. IX. S. 1215. Rheuter, Die Behandlung der pelvinen Dystokien, bes. die Einleitung der künstlichen Frühgeburt in der Geburtshilflichen Klinik in Lyon 1904-1920. Gyn. obstet, VI. 1. S. 10. Rißmann, Die Stoffwechselstörungen der Schwangerschaft, mit besonderer Berücksichtigung des Mineralstoffwechsels. Z. f. G. 26. VIII. S. 1376. Runge, H., Beitrag zur Frage des Eiidothelsymptoms in Geburtshilfe und Gynäkologie. Z. f. G. 12. VIII. S. 1303. Runge, E., Praktische Fragen der Cieburtshilfe. Med. Klin. 17. IX. S. 1226 und 24. IX. S. 1257. Sachs, Die Behandlung der Wehenschwäche. Med. Klin. 13. VIII. S. 1045. Sachs, Über die Behandlung einiger Anomahen der austreibenden Kräfte.

Klin. Woch. 9. IX. S. 1843. Samuel, Gefahren bei Anwendung der Hypophysenpräparate. Klin. Woch.

9. IX. S. 1839.

Literatur-Verzeichnis. 241

Schickele, Betrachtungen über· Anfang und Dauer der Schwangerschaft.

Gyn. Obstét. V. 6. S. 590. Schmitt, Zur Frage der Abortbehandlung. Munch, med. Woch. 25. VIII.

S. 1250. Schneider, M., Zur Behandlung des septischen Abortes. Med. Klin. 24. IX.

S. 1254. Schoenholz, Tubenhämatom bei intrauteriner Gravidität. T. f. G. 16. IX.

S. 1476. Schumann, Organische Nervenkrankheit als Schwangerschaftskomplikation.

Bericht über 2 Fälle. Am. J. obstet. gyn. IV. 1. S. 67. Schwab, Die Ursache des unstillbarenErbrechens in der Schwangerschaft.

Z. f. G. 19. VIII. S. 1343. Schweilzer, Klinisches und patliologisch-anatomisches zur Blasenmole und

Chorion epithelioma malignum. Med. Klin. 27. VIII. S. 1113. Sel·,d, Omnadin (Immunovakzin) bei- akuten Infektionen. Munch, med.

Woch. 18. VIII. S. 1110. Sellheim, Erklärung zur Achsendrehung der inneren Organe sowie dio Drehung, Umschlingung und Verknotung der Nabelschnur. Munch.

med. Woch. 25. VIII. 22. .S1/8ori, PuerperaleGassepsisuncllkterus. Munch, med. Woch. 18. VIII. S. 1209. Sippel, Heterotope Zwillingsschwangerschaft im Uterus und Tube mit nachfolgender Tubenschwangerschaft in der anderen Seite. Dtsch.

med. Wöch. 8. IX. S. 1202. Sir, Netzhautblutungen in der Gravidität. Casopis lék. cesk. 1921.

Nr. 51. Strakosch, Geburtshilfliches für die Praxis. Die Wendung. Med. Klin.

13. VIII. S. 1065. Sträuli, Sectio caesarea transperitonealis bei akutem Lungenödem.

Zwillingsschwangerschaft bei schwerem Vitium (,'ordis. Schweiz. med. Woch.

1921. Nr. 11. Temewárg, Zur hundertsten Jahreswende der Entdeckung der fötalen

Herztöne. Orvosi het. 21. Nr. 52. Vogt, Die -Bedeutung des großen Xetzes für das Schicksal ektopischer

Früchte. Z. f. Geb. u. Gyn. LXXXIV. 1. S. 152. Walthard, B., Zur Leberfunktion sub partu. Z. f. G. 12. VIII. S. 1301. Waren, ZweiFälle von Kaiserschnitt. Acta soc. med. fenencai XII. I. III. 
fan. 1 u. 2. Williams, Ph., Die Phenoltetrachlorphthaleinprobe der Leberfunktion während der Schwangerschaft. Am. J. obst. gyn. IV. 1. S. 26. Welz und van Nest, Beobachtungen über die Syphilisbehandlung im Gesundheitsamt in Detroit. Am. J. obst. gyn. IV. 2. S. 174. Wohlwill, Traumatische Geburtsschädigung des Gehirns. Munch, med.

Woch. 25. VIII. S. 1256. Wright, Die Ansichten primitiver Völker über Abort. Am. J. obst. gyn. IV. 2. S. 202. Zangemeister, BeitragzurLehrevom engenBecken. Z. f. Gyn. 2. IX. S. 1395.

Zarate, Die uterine Dystokie: Xotwendigkeit einheitlicher Nomenklatura

Gyn. obstet. VI. 1. S. 1. Zondek, Tiefenthermometrie (VII. Mitteilimg). Über Tiefenwirkung in der physikalischen Therapie. Munch, med. Woch. 21. IV. S. 579.

242

Literatur Vorzeicliiiis.

Gynäkologie.

Andresen, Reflexsymptome der Erkrankung der vreiblichen Beckenorgane.

Am. J. obst. Gyn. IV. S. 142. Asch und Wolff, Diagnose und Behandlung der Gonorrhöe des Weibes

und die Feststellung ihrer Heilung. Munch, med. Woch. 1. IX.

S. 1272 u. 8. IX. S. 1310. Burkhard, Ein Fall von Echinococcus hydatidicus in einer Mamma lactans.

Munch, med. Woch. 25. VIII. S. 1255. Byford, Einfachere Apparate zur Bluttransfusion nut der Zitrat-Methode.

Surg. gyn. obst. Aug. 22. S. 24. Coley, Pulsatilla bei Dysmenorrhöe. Brit. m. J. Xr. 3184. Cooke Hirst, Einige diskutable Punkte der Diagnose von Ursachen und

Behandlung der Sterilität. Am. J. obst, Gyn. IV. 2. S. 160. Dickinson, Xachweis der

Duchgängigkeit der Tuben durc $\cdot h$ eine laftgefüllte

Pipette. Am. J. bost. gyn. IV. 2. S. 159. Engelmann, Varikozele des Lig. lat. der Varicucele pelvica ? Z. f. Gyn.

26. VIII. S. 1374. van Etíen, Über 75 aufeinanderfolgencle Fälle von Hysterektomie wegen Uterusfibroms. Am. J. obst. gyn. IV. 2. S. 169. Eunike, Isoliert torquierte normale Tube. Z. f. Gyn. 16. IX. S. 1484. Fecher, Zur instrumentellen Knotenbildung. Munch, med. Woch. 18. VIII. S. 1217. Frank1, Zur Beurteilung der Qualität des Karzinommaterials. Z. f. G.

12. VIII. S. 1300. Geísí u. Somberg, Präoperative Digitalisdarreichung: eine Methode postoperative Komplikationen zu vermeiden. Am. J. obst. gyn. IV. 2.

S. 135. Graf, Zur operativen Behandlung der Sterilität. Wien. klin. Woch. 31. VIII.

S. 721. Hasdhorst, Praktische Brauchbarkeit der Senkungsprobe der Eiythrozyteu

in der Gynäkologie nebst Untersuchungen über Feblerquellen des

Verfahrens. Dtsch. med. Woch. 18. VIII. S. 1100. Hastrup, Ventrofixatio uteri. Zbl. f. Gyn. 19. VIII. S. 1341. Haub, Inversion der Harnblase bei Carcinoma vaginae et uteri. Munch.

med. Woch. 25. VIII. S. 1254. Haub, Untersuchungen über Hypertonie im Klimakterium.

Munch, med.

Woch. 25. VIII. S. 1256. Hirsch, M., Die Uterussonde ist ein gefährhches und entbehrliches Instrument, Z. f. G. 9. IX. S. 1442. Kehrer, Ursacheii vmd Behandlung der Unfruchtbarkeit nach modernen

Gesichtspunkten. Dresden und Leipzig, Ih. Steinkopff. 1922. Keller, Studie über die Zysten des gelben Körpers. Gyn. obstet. V. 6. S. 458. Klauser, Blasenvorfall durch die Harnröhre. Munch, med. Woch. 15. IX. 
S. 1344. Körner, Die cperative Behandlung der Retroflexio uteri mittels Ligamentoventrofixatio. Z. f. Geb. u. Gyn. LXXXIV. 1. S. 164. Kreis, Ivlinische Untersuchungen über den Tonus des Sympathikus und

Parasympathikus in Beziehuna zur Menstruation, bes. der Amenorrhöe.

Gyn. obstet, V. 6. S. 543.

Literatur-VerzeicUniá. 243

Kroß, Wirkung der Injektion des Extrakts der Vorderlappen der Zirbeldrüse auf die Generationsorgane. Am. J. obst. gyn. IV. 1. S. 19. Lieben, Ein Fall von Kompression der Vena iliaca durcli exzessiv gedehntes

Zoekum. Med. Klin. 3. IX. S. 1148. Loewenstein und Schapiro, Behandlung des gonorrhöischen Fluor mittels

Z\%orscher Stauung und Vakzinebehandlung. Med. Klin. 3. IX. S. 1147. Mackinney, Unvermutete männliche Sterilität. Am. J. obst. gyn. IV. 2.

S. 165. Masson, Über nicht-teratoide Ovarialtumoren. Gynéc. obst. VI. 2. S. 81. Maümüller, Beitrag zur Statistik der Genitalkarzinome. Z. f. Geb. u. Gyn.

LXXXIV. 1. S. 106. Meleneg, Syncytioma (atypisches Chorioma) uteri. Ausgang in akute Peritonitis. Surg. gyn. obst. Aug. 22. S. 137. Menge, Zur Therapie der Dysmehorrhöe. Z, f. Gyn. 19. VIII. S. 1330. Meyer, K., Über den Einfluß der Menstruation auf die Chronizität der eitrigen Endometritis. Schweiz. med. Woch. 1921. Nr. 42. Miller, J. W., Über die Varikozele des Ligament-urns latum. Z. f. G. 26. VIII.

S. 1370. Neurath, Die Geschlechtsreife und ihre Pathologie. Wien. klin. Woch.

21. IX. S. 731. Oeiíingen, v., Über den Einfluß der Plasmastruktur auf das Zelleben. Z.

f. Gyn. 9. IX. S. 1431. Oil, v., Die Entwicklung der Hysteromyomektomie. Gynéc. obst. VI. 2.

S. 106. Roysler, Marion Sims. Chirurgische Meister von Amerika. Surg. obst. Gyn. Aug. 22. S. 237. Salomon und Rath, Die Entstehung der Genitalflora. Z, f. Geb. u. Gyn. LXXXIV. 1. S. 141. Scheid, Weiterer Beitrag zur Stieldrehung der Adnexe im kindlichen Alter. Z. f. G. 16. IX. S. 1485. Schickelé, Studien über die Funktion der Eierstöcke (III. Teil). - Der primäre mucipare glanduläre Eierstockskrebs. - Gyn. obstét. V. 6.

S. 425 u. 446. Schubert, d., Über Operationssaalbeleuchtung. Z. f. Gyn. 9. IX. S. 1437. Schroder, Rob, Zur Analyse der genitalen Blutungen bei nichtschv $1 / 8 \cdot$ angeren

Frauen. Klin. Woch. 23. IX. S. 1929. Scott, Leukozytose nach gynäkologischen Operationen. 50 Beobachtungeii

mit Bericht über zeitweilige postoperative Lei $1 / 80 z y$ tenzählung.

Surg. gyn. obst. August 22. S. 181. Sieveking, Die Zunahme der Geschlechtskrankheiten in deutschen Groß-

städten, ihre Gefahren und die Möglichkeit ihrer Abwehr. Klin. Woch.

30. IX. S. 2005. Sippel, Die postoperative Kochsalzinfusion in aie Bauchhöhlc. Z. f. G.

16. IX. S. 1474. Stajano, Die phrenische Reaktion und die Genitalinfektion. Gyn. obst.

VI. 1. S. 44. Stickel und Zondek, Klinische Untersuchungen über den Wert der Organo therapie bei ovariellon Blutuirgen. Z. f. Geb. u. Gyn. LXXXI $\tau$. 1.

S. 83.

244 Literatur-Verzeichnis.

Síoeckel, Uterusperforation mit schwerer Verletzung der Adnexe. Z. f. G.

2. IX. S. 1410. Stríiver, Die Mechanik des Iubenverschlusses und ihre Bedeutung für die Pathogenese der Tuben-Ovarialzysten. Z. f. Geb. u. Gyn. LXXXIV. 1.

S. 58. Talboí, Versuch, clironische Sepsis während der Schwangerschaft zu heilen. 
Surg. gyn. obst. Aug. 22. S. 187. Wolff, F., Hat der Krieg die Operabilität des Uteruskrebses verschlechtert?

Z. f. G. 19. VIII. S. 1341. Zangemeister, Rückblick auf den Gynäkolcgenkongreß. Z. f. G. 12. VIII.

S. 1298. Zondek, ïiefenwirkung bei thermischer Behandlung. Med. Klin. 26. VIII.

S. 1744.

Strahlentherapíe.

Flatau, Die Heilung klimakterischer Gebärmutterblutungen durch Radium.

Munch, mecl. Woch. 1. IX. S. 1277. FIaskamp, Röntgenschädigungen, ihre zivil- und strafrechtlichen Folgen.

Klin. Woch. 23. IX. S. 1954. Fraenkel, Manfred, Zxvc ïheorie der zellfunktionerhöhenden Röntgen-

strahlen. Dtsch. med. Woch. 25. VIII. S. 1136. Frankl, Über das Wachstum implantierter Karzinome bei Vorbehandlung

mit Röntgenstrahlen. Wien. klin. Woch. 31. VIII. S. 726. Haeger, Eine neue

Beleuchtungsemrichtung für Röntgenzimmer. Munch.

med. Woch. 21. IV. S. 594. Kolischcr, Chirurgische Diathermie- und Radiotherapie bei Uteruskrebs.

Surg. Gyn. Obst, Aug. 22. S. 227. Jilahnert, Über den Einfluß der Röntgenbestrahlung auf das Kohlensäure-

bindungsvermögen des Blutes. Klin. Woch. 9. IX. S. 1840. Meyer, M., Ein Beitrag zur Frage der Spätreaktion nach Röntgenbestrah-

lungen. Z. f. G. 12. VIII. S. 1315. Recasens, Die Röntgentherapie in der Gynäkologie. Madrid, J. Cosano. 1921. Schugt, Kapillarmikroskopie des Röntgenerythems an der Bauchhaut.

Munch, med. Woch. 11. VIII. S. 1178. Tsukahara, Experimentelle Untersuchungen über die Beeinflussung der inneren Sekretion des Ovariums durch Röntgenstrahlen. Z. f. Geb.

u. Gyn. LXXXIV. 1. S. 36. 